\title{
Cancer, pre-cancer and normal oral cells distinguished by dielectrophoresis
}

H.J. Mulhall $^{(1)}$, F.H. Labeed ${ }^{(1)}$, B. Kazmi ${ }^{(2)}$, D.E. Costea ${ }^{(4)}$, M.P. Hughes ${ }^{(1)}$ and M.P. Lewis $^{(2,3, *)}$

${ }^{(1)}$ School of Biomedical Engineering, University of Surrey, Guildford, Surrey, England GU2 7XH

${ }^{(2)}$ UCL Eastman Dental Institute, 256 Gray's Inn Road, London, England WC1X 8LD

${ }^{(3)}$ Muscle Cellular and Molecular Physiology Research Group, ISPAR, University of

Bedfordshire, Polhill Avenue, Bedford MK41 9EA.

${ }^{(4)}$ Department of Pathology, The Gade Institute, University of Bergen, Haukeland Hospital, N-5021 Bergen, Norway.

*Corresponding author.

Running Title: Oral Cancer and DEP 


\section{Abstract}

Most oral cancers are oral squamous cell carcinomas (OSCC) that arise from the epithelial lining of the oral mucosa. Given that the oral cavity is easily accessible, the disease lends itself to early detection; however most oral cancers are diagnosed at a late stage and approximately half of oral cancer sufferers do not survive beyond 5 years, post-diagnosis. The low survival rate has been attributed to late detection but there is no accepted, reliable and convenient method for the detection of oral cancer and oral pre-cancer.

Dielectrophoresis (DEP) is a label-free technique which can be used to obtain multiparametric measurements of cell electrical properties. Parameters, such as cytoplasmic conductivity and effective membrane capacitance $\left(\mathrm{C}_{\mathrm{Eff}}\right)$, can be non-invasively determined by the technique. In this study, a novel lab-on-a-chip device was used to determine the cytoplasmic conductivity and $\mathrm{C}_{\mathrm{Eff}}$ of primary normal oral keratinocytes, and pre-cancerous and cancerous oral keratinocyte cell lines.

Our results show that the electrical properties of normal, pre-cancerous and cancerous oral keratinocytes are distinct. Furthermore, increasing $\mathrm{C}_{\mathrm{Eff}}$ and decreasing cytoplasmic conductivity correlate with disease progression which could prove significant for diagnostic and prognostic applications. DEP has the potential to be used in a primary health care setting as a non-invasive and non-subjective technique to detect oral cancer and oral precancer. 
Keywords

OSCC; Oral Cancer; Dielectrophoresis; DEP; Diagnostics; Detection. 


\section{Introduction}

Oral cancer is the sixth most common cancer, worldwide [1]. In countries such as Pakistan, Bangladesh, India and Sri Lanka, oral cancer is the most common cancer and accounts for up to $30 \%$ of all diagnosed cancers [1]. Ninety per cent of oral cancers are oral squamous cell carcinoma (OSCC) [2]. Given that the oral cavity is easily accessible, the disease lends itself to early detection. However, the survival rate for oral cancer is lower than those for breast, colorectal, prostate and cervical cancers [3] and late stage diagnosis has been recognized as a contributing factor [4, 5]. Currently, pre-malignant and malignant oral lesions are identified by conventional oral examination (COE) which comprises of the visual inspection and palpation of the oral cavity [2]. Often manifesting with features synonymous with common benign oral lesions, they are difficult to discern impeding COE screening [6-9]. Oral lesions are common [10-13] and most are benign [14, 15]. The goldstandard diagnostic technique is invasive, painful, requires the use of anaesthetic and can result in complications such as infection. Consequently, only a small number of lesions are investigated by the gold-standard method and early stage or innocuous appearing malignant lesions are often overlooked. A non-invasive, non-subjective pre-OSCC and OSCC detection method that can be carried out in a primary health care setting would be of great benefit.

The transition of a cell from a healthy to pathological state is associated with subsequent variation in its electrical properties [16-20] indicating the potential for electrophysiological markers to be used in the detection and diagnosis of disease. Recently, it has been found that the electrical properties of malignant breast epithelial cells are different to quasinormal breast cells [20] and malignant oral epithelial cells are different to quasi-normal dermal epithelial cells [21] suggesting a use for electrophysiological markers in the detection and diagnosis of carcinomas.

Yang et al. [22] showed that an OSCC cell line could be distinguished from an immortalised, non-cancer-derived oesophagus cell line by electrical impedance-based 
measurements. The group demonstrated that cellular activity such as adherence and proliferation induced changes in the capacitance and resistance of the electrode to which cells were adhered to. The kinetics of the two cell lines were distinct, indicating that electrical impedance-based methods could be used in the early diagnosis of oral cancer. However, this method relies on harvesting adherent cells from the oral mucosa and the measurements take several hours. The present study has employed an AC electrokinetic technique called dielectrophoresis (DEP) to determine the electrical properties of normal, pre-OSCC (dysplastic) and OSCC oral epithelial (keratinocyte) cells. DEP is performed when cells are in suspension, so cell adhesion is not required for measurements. This allows for non-invasive cell harvesting methods such as exfoliation with a brush biopsy tool. In addition, results can be acquired in as little as 30 seconds [23].

The cell's ability to conduct electric charge (conductivity) and to store electric charge (capacitance) can be measured using DEP. DEP is a phenomenon whereby a cell is induced to move under the influence of a non-uniform electric field [24]. When a non-uniform electric field is applied to a cell in suspension, the cell becomes polarised. The electric charges on the surface of the cell interact with the applied electric field and forces are exerted on the cell. If the electric field strength is spatially uniform, the forces will be equal and the cell will remain stationary. However, if the electric field strength is non-uniform then there will be an imbalance in forces and the cell will move. The direction and velocity of cell motion is dependent on the electrical properties of the cell and its suspending medium, and the frequency of the applied electric field. By observing cell motion over a range of frequencies the cells' electrophysiological spectrum or 'fingerprint' can be determined. Multi-parametric electrical data can be obtained from the cell's electrophysiological fingerprint using a single-shell model $[25,26]$.

This study has used a novel DEP-microwell electrode system [27, 28] to determine the electrical properties of primary normal oral cells and dysplastic and OSCC oral keratinocyte cell lines. The DEP-microwell system has been shown to be rapid, easy to use 
and low cost and has the potential to be automated and integrated into bench-top apparatus situated in a primary health care setting, such as a dental surgery. As spectra can be collected in as little as 30 seconds [23], waiting times for laboratory tests would be eliminated. Automation of the device would render the results free from subjective assessment allowing clinicians with minimal training and expertise to utilize the technique effectively. It is proposed that DEP can be used in a primary health care setting as a cheap, non-invasive OSCC detection system.

\section{Materials and Methods}

\subsection{Cell Culture}

The OSCC cell lines H357 and H157 were a kind gift from Dr. S.A. Whawell, University of Sheffield, U.K. H357 and H157 cells were cultured in keratinocyte growth medium (KGM) which consisted of 3 volumes of low glucose $\left(1 \mathrm{~g} \mathrm{~L}^{-1}\right)$ DMEM (PAA, Somerset, UK) and 1 volume of F12 Hams (Invitrogen, Paisley, UK). The basal medium was supplemented with $10 \%$ foetal calf serum (FCS), $10^{-10} \mathrm{M}_{\text {cholera toxin, } 0.5 \mu \mathrm{g} \mathrm{ml}^{-1}}$ hydrocortisone, 10ng ml${ }^{-1}$ epidermal growth factor (EGF), $5 \mu \mathrm{g} \mathrm{ml}^{-1}$ insulin, $2.5 \mu \mathrm{g} \mathrm{ml}^{-1}$ amphotericin $\mathrm{B}, 1.8 \times 10^{-4} \mathrm{M}$ adenine, $100 \mathrm{U} \mathrm{ml}^{-1}$ penicillin and $100 \mu \mathrm{g} \mathrm{ml}^{-1}$ streptomycin. Primary Human Oral Keratinocytes (HOK) were purchased from TSC Cellworks Ltd. (Buckingham, U.K.) HOK cells were grown in Oral Keratinocyte Medium (OKM) (TSC Cellworks Ltd., Buckingham, U.K.). Dysplastic Oral Keratinocyte (DOK) cells were purchased from the European Collection of Animal Cell Cultures (ECACC). DOK cells were grown in DMEM supplemented with $10 \% \mathrm{FBS}, 5 \mu \mathrm{g} \mathrm{ml}^{-1}$ hydrocortisone, $100 \mathrm{U} \mathrm{ml}^{-1}$ penicillin and $100 \mu \mathrm{g} \mathrm{ml}^{-1}$ streptomycin. DMEM and FCS were purchased from PAA (Somerset, UK). EGF was purchased from ProSpec-Tany Technogene Ltd. (Rehovot, Israel). All other products were purchased from Sigma Aldrich (Poole, UK). All cells were cultured in a humidified incubator at $37^{\circ} \mathrm{C}$ with $5 \% \mathrm{CO}_{2}$.

\subsection{Sample Preparation}

DEP experimental medium was prepared containing $17 \mathrm{mM}$ glucose and $263 \mathrm{mM}$ sucrose in deionised water $[29,30]$. The medium was adjusted to a conductivity of $5 \mathrm{mS} \mathrm{m}^{-1}$ by 
addition of PBS and verified with a Jenway 470 conductivity meter. To confirm that DEP experimental medium was isosmotic with respect to cell growth medium, DEP experimental medium was measured using an Osmomat 030 (Ganotec) osmometer (Wolf Laboratories Limited, York, UK). Cells were washed twice by centrifugation at 100xg and resuspended in DEP experimental medium at a cell concentration of $2 \times 10^{6}$ cells $\mathrm{ml}^{-1}$.

\subsection{Dielectrophoresis Experiments}

The DEP-microwell electrode system was used to determine the electrophysiological properties of oral keratinocytes in this study. The fabrication of the DEP-microwell and experimental methods are described in detail elsewhere [27, 28, 31]. Briefly, for each experiment, cell motion was measured in response to a non-uniform AC electric field for five frequencies per decade from $4 \mathrm{kHz}$ to $20 \mathrm{MHz}$. A MatLab (The Mathworks Inc, Nantick USA) script was used to assess the change in light intensity in the DEP-microwell over the duration of time that the electric potential was applied. The change in light intensity was normalised to an image captured before the electric potential was applied. The electrophysiological properties were derived by fitting a 'single-shell' model to the line of best fit through the electrophysiological spectra $[17,26]$. The line of best fit had a correlation co-efficient of 0.9 or above for all experiments. Cell radii were obtained by capturing images of at least 50 cells per experiment on a haemocytometer and measuring the cell diameter using image analysis software.

\subsection{Polarisation and Dielectrophoresis Theory}

A dielectric is a material which has the capacity to polarise when subject to an applied electric field [32]. Polarisation is the redistribution of charges bound within a material under the influence of an applied electric field, whereby charges of the same magnitude and opposite polarity are displaced to different locations within the material. On removal of the applied electric field, the material returns to its original state. Polarisation is not instantaneous; charges take a finite amount of time to accumulate. Following removal of the applied electric field, a finite amount of time is taken for charge equilibrium to be reestablished. This is termed relaxation time $(\tau)$. 
In systems where an alternating current (AC) is used to produce an electric field, polarisation is frequency dependent. At low frequencies, polarisation realizes its maximum potential before the direction of the electric field changes. Redistribution of free charge (conductivity) is the dominating mechanism governing polarisation at low frequencies. At high frequencies, polarisation does not have time to realize its maximum potential before the direction of the field changes. Perturbation of bound charges (permittivity) dominates polarisation at high frequencies. The difference between the two states is called a dielectric dispersion.

When a dielectric particle is suspended in a conductive medium and an electric field is applied, the particle will become polarised. When the particle is more polarisable than the suspending medium, the charge density at the internal aspect of the interface between the particle and the suspending medium is greater than the charge density at the external aspect of the interface. Thus, the net induced dipole is aligned with the electric field and the electric field lines will bend towards the particle meeting its surface at right angles. When the particle is less polarisable than the suspending medium, the charge density at the internal aspect of the interface between the particle and the suspending medium is less than the external aspect of the interface. Thus, the net induced dipole aligns counter to the electric field and the electric field lines will bend around the particle as if it were an insulator. When the charge density at the internal aspect of the interface between the particle and the suspending medium is equal to the external aspect of the interface, the polarisability of the particle and medium will be equal and the applied electric field remain unperturbed.

The interaction between the separated charges on each side of the induced dipole and the applied non-uniform electric field generates a force. When a dielectric particle is suspended in a conductive medium and a spatially uniform electric field is applied, the magnitude of the force imparted on the two poles of the induced dipole is equal and opposite. Thus, the net force acting on the particle is zero and the particle remains stationary. 
When the applied electric field is spatially non-uniform, a gradient of electric field strength exists. When a dielectric particle is suspended in a conductive medium and a spatially nonuniform electric field is applied, the strength of the electric field on one pole of the dipole is greater than on the other. Therefore, there is an imbalance of Coulomb forces imparted on the induced dipole. Thus, a translational force is induced and the particle will undergo lateral displacement. The polarisability of the particle relative to that of the suspending medium governs the direction of particle motion. If the particle is more polarisable than the suspending medium, the particle will move in the direction of high field strength regions. This is termed positive DEP (+DEP, p-DEP). If the particle is less polarisable than the suspending medium the particle will be repelled from high field strength regions. This is termed negative DEP (-DEP, n-DEP). If the polarisability of the particle is equal to that of the medium, the particle will remain stationary; the frequency at which this occurs is termed the crossover frequency $\left(\mathrm{F}_{\mathrm{CROSS}}, \mathrm{F}_{\mathrm{C}}, \mathrm{F}_{\mathrm{X}}\right.$ or $\left.\mathrm{F}_{\mathrm{X} 0}\right)$.

The magnitude of particle displacement is proportional to the force exerted on the particle. The DEP force is given by Equation 1:

$$
F_{D E P}=2 \pi r^{3} \varepsilon_{m} \operatorname{Re}[K(\omega)] \nabla E^{2}
$$

Equation 1

Where $r$ is the radius of the particle, $\varepsilon_{m}$ is the medium permittivity, $\nabla$ is the Del operator (gradient) on the applied electric field $E$ and $\operatorname{Re}[K(\omega)]$ is the real part of the ClausiusMossotti factor. The Clausius-Mossotti factor is given by Equation 2:

$$
K(\omega)=\frac{\varepsilon_{p}^{*}-\varepsilon_{m}^{*}}{\varepsilon_{p}^{*}+2 \varepsilon_{m}^{*}}
$$

Equation 2

Where $\varepsilon_{p}{ }^{*}$ and $\varepsilon_{m} *$ are the complex permittivities of the particle and medium, respectively. The complex permittivity is given by Equation 3: 


$$
\varepsilon^{*}=\varepsilon-\frac{j \sigma}{\omega}
$$

Equation 3

Where $\boldsymbol{\varepsilon}$ is the permittivity, $\boldsymbol{\omega}$ is the angular frequency, $\boldsymbol{j}$ is the square root of -1 and $\boldsymbol{\sigma}$ is the conductivity. Thus, the permittivity and conductivity of the particle can be derived from measurements of cell motion as a function of frequency.

Homogenous dielectric particles have a single dielectric dispersion caused by the accumulation of charges at the interface between the suspending medium and the particle. The DEP force expression (Equation 1) holds true for the dielectrophoretic force of a homogeneous sphere. A biological cell is not homogenous; it is an anisotropic dielectric consisting of a membrane and a cytoplams, each of which has discrete polarisabilities.

Multi-shelled models are used to interpret dielectric spectroscopy of cells [17, 25, 26, 31]. The most common approach divides the model into concentric spheres with increasing radii. Each sphere is a homogenous particle suspended in a medium that has the properties of the next biggest sphere. The simplest form of the model, a 'single-shell' model, is an approximation of the structure of a mammalian cell whereby the cell is divided into a homogenous core, representing the cytoplasm, surrounded by a shell representing the plasma membrane.

The effective complex permittivity for the entire particle is a combination of the properties of each individual sphere. The effective complex permittivity for the inner and outer spheres can be represented by a single expression combining the properties of each, effectively removing the concentric sphere from the model and replacing it with a combined shell. This is given by Equation 4 . 


$$
\varepsilon_{1 \varepsilon f f}^{*}=\varepsilon_{2}^{*} \frac{\left(\frac{r_{2}}{r_{1}}\right)^{3}+2\left(\left(\varepsilon_{1}^{*}+\varepsilon_{2}^{*}\right) /\left(\varepsilon_{1}^{*}+2 \varepsilon_{2}^{*}\right)\right)}{\left(\frac{r_{2}}{r_{1}}\right)^{3}-\left(\left(\varepsilon_{1}^{*}-\varepsilon_{2}^{*}\right) /\left(\varepsilon_{1}^{*}+2 \varepsilon_{2}^{*}\right)\right)}
$$

Equation 4

The subscript eff refers to the effective complex permittivity. Subscripts $\mathbf{1}_{1}$ and ${ }_{2}$ refer to the core and the shell, respectively, and $r_{1}$ and $r_{2}$ correspond to the radius of the core and the radius of the centre of the sphere to outside of the shell. Thus the Clausius-Mossotti factor is given by Equation 5 .

$$
k(\omega)=\frac{\varepsilon_{1 \text { eff }}^{*}-\varepsilon_{3}^{*}}{\varepsilon_{1 \text { eff }}^{*}+2 \varepsilon_{3}^{*}}
$$

Equation 5

The subscript 3 corresponds to the suspending medium. Thus, the conductivity and permittivity values for the membrane and cytoplasm can be determined by curve fitting the model to the data.

The effective membrane capacitance $\left(\mathrm{C}_{\mathrm{EFF}}\right)$ is given by Equation 6 .

$$
C_{E F F}=\frac{\left(\varepsilon_{2} s_{0}\right)}{\left(r^{2}-r^{1}\right)}
$$

Equation 6

The $\mathrm{C}_{\mathrm{EFF}}$ is a function of the permittivity and thickness of the cell membrane. It has been estimated that a completely smooth cell membrane has a value of approximately $6 \mathrm{mF} \mathrm{m}^{-2}$ [33]. The $\mathrm{C}_{\mathrm{EFF}}$ assumes a sphere with a smooth membrane so an increase in surface area by means of membrane folding would result in an increase in the $\mathrm{C}_{\mathrm{EFF}}$ value. Therefore, it follows that the $\mathrm{C}_{\mathrm{EFF}}$ is also a function of surface morphology. High values of $\mathrm{C}_{\mathrm{EFF}}$ correlate with a high number of blebs, folds, ruffles and microvilli in the cell membrane [34-36]. 


\subsection{Statistical Analysis}

Statistical analysis was carried out using SPSS version 16.0 (SPSS Inc. Chicago, IL). Cell electrophysiological properties and radius measurements were analysed graphically using a histogram to determine whether the distribution conformed to a normal curve. If the distribution was normal, the parametric two independent sample student's t-test was used. If normality could not be confirmed, the non-parametric, two independent sample Mann Whitney-U test was performed. Analysis was two-way and $P$ values displayed are of the exact variant. $P$ values of $<0.05$ were considered statistically significant.

\section{Results}

When energised with a current, the cell suspensions in the DEP-microwell responded by being attracted to, or repelled from, the electrodes at the perimeter of the DEP-microwell, as shown in Figure I. The direction of cell motion was dependent on the frequency of the applied electric field. Changes in light intensity in regions of the well close to the electrode edges are proportional to changes in cell concentration [27]. By probing cell samples over a frequency range, electrophysiological spectra with negative and positive domains were produced, as shown in Figure II.

The DEP-microwell was used to determine electrophysiological spectra of primary normal oral keratinocytes (HOK) and dysplastic (DOK) and malignant (H357 and H157) oral keratinocyte cell lines. The single-shell model was used to determine the effective membrane capacitance (capacitance per unit area of the membrane) $\left(\mathrm{C}_{\mathrm{Eff}}\right)$ and the cytoplasmic conductivity from electrophysiological spectra of each cell type. The effective $\mathrm{C}_{\mathrm{Eff}}$ values of HOK, DOK, H357 and H157 cells were found to be 0.69 ( \pm sd 0.06$), 1.09$ ( \pm sd 0.2), 1.51 ( \pm sd 0.26) and 1.43( \pm sd 0.45) $\mu \mathrm{F} \mathrm{cm}{ }^{-2}$, respectively. DOK, H357 and H157 all had higher mean effective $\mathrm{C}_{\mathrm{Eff}}$ values than HOK cells $(\mathrm{P}=0.008, \mathrm{P}=0.008$ and $\mathrm{P}=0.008$, respectively), see Figure III. H157 cells had a higher mean effective $\mathrm{C}_{\text {Eff }}$ values than DOK cells $(\mathrm{P}=0.048)$. The cytoplasmic conductivities of $\mathrm{HOK}, \mathrm{DOK}, \mathrm{H} 357$ and $\mathrm{H} 157$ were found to be $0.71( \pm \mathrm{sd} 0.08), 0.42( \pm \mathrm{sd} 0.26), 0.26( \pm \mathrm{sd} 0.06)$ and $0.25( \pm \mathrm{sd} 0.10) \mathrm{S} \mathrm{m}^{-1}$, respectively. $\mathrm{H} 357$ and $\mathrm{H} 157$ cells all displayed lower mean cytoplasmic conductivities 
than HOK cells $(\mathrm{P}=0.008$ and $\mathrm{P}=0.008$, respectively), see Figure IV. Although not statistically significant, H357 and H157 had lower mean cytoplasmic conductivities than DOK cells $(\mathrm{P}=0.548$ and $\mathrm{P}=0.222$, respectively). The DEP scatter plot in Figure V shows cytoplasmic conductivity as a function of effective $\mathrm{C}_{\mathrm{Eff}}$. HOK cells occupy an area that is distinct to DOK, H157 and H357 cells in the upper left region of the graph. While DOK, H157 and H357 cells occupy overlapping regions, the cancerous cells (H157 and H357) occur in a cluster in the lower region of the graph shifted toward the right and dysplastic cells (DOK) occur in a cluster occupying the central region of the graph.

\section{Discussion}

With a view to improving the early detection rate of oral cancer, this investigation sought to assess the potential for DEP to be used as a tool in the detection of dysplastic and cancerous epithelial cells in the oral cavity. The effective $\mathrm{C}_{\mathrm{Eff}}$ and cytoplasmic conductivity of primary normal oral keratinocytes (HOK) and dysplastic (DOK) and cancerous (H357 and H157) oral keratinocytes have been determined. The mean effective $\mathrm{C}_{\mathrm{Eff}}$ values of DOK, H357 and H157 were higher than HOK cells $(\mathrm{P}=0.008, \mathrm{P}=0.008$ and $\mathrm{P}=0.008$, respectively). The mean effective $\mathrm{C}_{\mathrm{Eff}}$ of $\mathrm{H} 357$ and $\mathrm{H} 157$ cells was higher than DOK cells $(\mathrm{P}=0.333$ and $\mathrm{P}=0.048$, respectively), although this was only statistically significant for H157 cells. The OSCC cell lines, H357 and H157, showed similar mean values for effective membrane capacitance $\left(1.51\right.$ and $1.43 \mu \mathrm{F} \mathrm{cm}^{-2}$, respectively).

The effective $C_{E f f}$ is a function of membrane surface area, permittivity and thickness. It has been estimated that a cell with a completely smooth membrane has a $\mathrm{C}_{\mathrm{Eff}}$ of approximately $0.6 \mu \mathrm{F} \mathrm{cm}^{-2}$ [33]. The effective $\mathrm{C}_{\mathrm{Eff}}$ determination assumes a sphere with a smooth membrane so an increase in surface area by means of membrane folding would result in an increase in the $\mathrm{C}_{\mathrm{Eff}}$ value. Therefore, the effective $\mathrm{C}_{\mathrm{Eff}}$ can be regarded as an indicator of surface morphology. High values of effective $\mathrm{C}_{\mathrm{Eff}}$ correlate with a high number of blebs, folds, ruffles and microvilli in the cell membrane [34-36]. Dysplastic oral cells have a 1.59fold (58.7\%) higher mean effective $\mathrm{C}_{\mathrm{Eff}}$ than normal oral cells. Cancerous oral cells have approximately a 1.35 -fold (38.4\% and $34.4 \%)$ higher mean effective $\mathrm{C}_{\mathrm{Eff}}$ than dysplastic 
oral cells and approximately a 2.14-fold (109.3\% and $119.6 \%)$ higher mean effective $\mathrm{C}_{\text {Eff }}$ than normal oral cells. The increase in mean effective membrane capacitance between normal, dysplastic and malignant cells indicates that the cell membrane morphology becomes increasingly ruffled with disease progression. This is consistent with observations that increased membrane ruffling is a component of the cancer cell phenotype and correlates with metastatic potential and invasiveness [37-39].

The mean cytoplasmic conductivity values of DOK, H357 and H157 cells were lower than HOK cells $(\mathrm{P}=0.135, \mathrm{P}=0.008$ and $\mathrm{P}=0.008$, respectively), although this was not statistically significant for DOK cells. The mean cytoplasmic conductivities of H357 and H157 cells were lower than DOK cells, although this was not statistically significant ( $\mathrm{P}=0.548$ and $\mathrm{P}=0.222$, respectively). The OSCC cell lines, $\mathrm{H} 357$ and $\mathrm{H} 157$, showed similar mean values for cytoplasmic conductivity (0.26 and $0.25 \mathrm{~S} \mathrm{~m}^{-1}$, respectively). DOK, H357 and H157 cells have a 40.8\%, 63.3\%, 64.7\% lower mean cytoplasmic conductivity than HOK cells, respectively. $\mathrm{H} 357$ and $\mathrm{H} 157$ cells have a $38.1 \%$ and $40.5 \%$ lower mean cytoplasmic conductivity than DOK cells, respectively. Differences in cytoplasmic conductivity could arise for reasons, including differences in the concentration of mobile ion species in the cytoplasms, differences in the mobility of mobile ion species in the cytoplasms differences in the membrane permeability of ionic and non-ionic osmolytes or differences in the intracellular volume fraction of macromolecules, organelles and proteins.

In reviewing the current literature and the results from this study, a trend has been observed; cells with a more malignant phenotype have a higher effective $\mathrm{C}_{\mathrm{Eff}}$ and a lower cytoplasmic conductivity than cells with a more normal phenotype. In a study by Gascoyne and colleagues, murine erythroleukemia cells showed a higher effective $\mathrm{C}_{\mathrm{Eff}}\left(1.26 \mu \mathrm{F} \mathrm{cm}{ }^{2}\right)$ than their counterpart cells which had undergone chemically induced differentiation $\left(0.88 \mu \mathrm{F} \mathrm{cm}^{2}\right)$ [19]. Becker and colleagues demonstrated that the cancerous leukocyte progenitor cell line HL-60 and the breast cancer cell line MDA231 have higher effective $\mathrm{C}_{\text {Eff }}$ values $\left(1.50 \mu \mathrm{F} \mathrm{cm}^{2}\right.$ and $2.60 \mu \mathrm{F} \mathrm{cm}{ }^{2}$, respectively) than normal $\mathrm{T}$ lymphocytes $(1.10$ $\left.\mu \mathrm{F} \mathrm{cm}^{2}\right)$ and erythrocytes $\left(0.90 \mu \mathrm{F} \mathrm{cm}^{2}\right)$ [40, 41]. Investigations of the temperature 
sensitive rat kidney cell line $6 \mathrm{~m} 2$ which possesses a normal phenotype at $39^{\circ} \mathrm{C}$ and a transformed phenotype at $33^{\circ} \mathrm{C}$ showed that cells in a transformed state had a higher effective $C_{E f f}$ than those in a normal state [17]. The amount of microvilli shown in scanning electron micrographs correlated with the effective $C_{\text {Eff }}$ values; transformed cells had increased microvilli compared normal cells. An et al.[20] found that normal breast cells (MCF 10A) had a higher cytoplasmic conductivity $\left(0.3 \mathrm{~S} \mathrm{~m}^{-1}\right)$ than their malignant counterparts (MCF 7) $\left(0.23 \mathrm{~S} \mathrm{~m}^{-1}\right)$. In the study by Broche et al.[21], which investigated the electrophysiological properties of keratinocyte cells, it was found that the quasi-normal dermal cell line, UP, had a lower effective $\mathrm{C}_{\mathrm{Eff}}$ than the OSCC cell line, H357. Furthermore, it was shown that UP cells had a higher cytoplasmic conductivity than H357 cells. This would suggest that for a number of cancers, DEP represents an alternative means of early detection; whilst it offers few advantages where the cancer is deep and needs to be surgically extracted for analysis, it presents many advantages for the detection of more superficially-cited cancers.

\section{Conclusion}

This investigation serves as a "proof-of-concept" study which demonstrates the potential for DEP to be used in the detection of oral cancer. Due to the accessibility of the oral cavity, DEP is a viable solution to the problem of OSCC detection. Furthermore, dysplastic oral keratinocytes have an electrophysiological phenotype distinct to cancerous oral keratinocytes which could prove significant in a diagnostic setting. Currently available OSCC detection techniques are subjective and show improved efficacy when employed by highly skilled and experienced clinicians $[42,43]$. However, these techniques are of most use in primary health care settings where handlers will be less experienced. The proposed DEP system is non-subjective, and does not require intensive sample preparation such as staining. It is proposed that DEP could be adapted to a bench top system that can be used for the detection of oral cancer by primary health care workers with minimal expertise and in resource limited settings. Further investigations with clinical samples are required to validate the assay. 
Oral cancer is a debilitating disease that has an extremely low survival rate in view of the accessibility of the oral cavity. A simple, low-cost, non-invasive detection technique to aid clinical assessment would be of great benefit. It has been shown that dysplastic and SCC oral keratinocytes can be identified on the basis of their electrophysiological phenotype; effective membrane capacitance and cytoplasmic conductivity correlates with disease progression. This work illustrates the potential for DEP to be used to detect a dysplastic or cancerous population of cells in the oral mucosa.

\section{Acknowledgements}

The authors wish to thank the Engineering and Physical Sciences Research Council for a scholarship for HJM. This work is dedicated to the memory of Mr. Brian Conroy, who was instrumental in initiating this research.

\section{Conflict of Interest}

The electrode design used is subject to a patent application by MPH.

\section{References}

1. Warnakulasuriya, S., Global epidemiology of oral and oropharyngeal cancer. Oral Oncology, 2009. 45(4-5): p. 309-316.

2. Silverman, S., Oral Cancer. Fifth Edition ed. 2003: B.C. Becker Inc.

3. Horner MJ, Ries LAG, Krapcho M, Neyman N, Aminou R, Howlader N, Altekruse SF, Feuer EJ, Huang L, Mariotto A, Miller BA, Lewis DR, Eisner MP, Stinchcomb DG, and (eds), E.B., SEER Cancer Statistics Review, 1975-2006. 2008, National Cancer Institute, Bethesda, MD. 
4. Silverman, S.J., Demographics and occurrence of oral and pharyngeal cancers: The outcomes, the trends, the challenge. The Journal of the American Dental Association, 2001. 132(suppl_1): p. 7S-11.

5. Al-Dakkak, I., Diagnostic delay broadly associated with more advanced stage oral cancer. Evidence-Based Dentistry, 2010. 11(1): p. 24-24.

6. Bagan, J., Sarrion, G., and Jimenez, Y., Oral cancer: Clinical features. Oral Oncology, 2010. 46(6): p. 414-417.

7. Silverman, S., Jr., Mucosal Lesions in Older Adults. The Journal of the American Dental Association, 2007. 138(suppl_1): p. 41S-46.

8. Silverman, S., Early diagnosis of oral cancer. Cancer, 1988. 62(S1): p. 1796-1799.

9. Shafer, W.G. and Waldron, C.A., Erythroplakia of the oral cavity. 1975. p. 1021-1028.

10. Sandler, H.C., Cytological screening for early mouth cancer. Interim report of the veterans administration co-operative study of oral exfoliative cytology. Cancer, 1962. 15(6): p. 1119-1124.

11. Burzynski, N., Firriolo, F., Butters, J., and Sorrell, C., Evaluation of oral cancer screening.

. Journal of Cancer Education, 1997. 12(2): p. 95-99.

12. Malaowalla, A.M., Silverman, S., Mani, N.J., Bilimoria, K.F., and Smith, L.W., Oral cancer in 57,518 industrial workers of Gujarat, India. A prevalence and followup study. Cancer, 1976. 37(4): p. 1882-1886.

13. Bouquot, J.E., Common oral lesions found during a mass screening examination. The Journal of the American Dental Association, 1986. 112(1): p. 50-57. 
14. Sciubba, J.J. and Collaborative Oral, C.S.G., Improving detection of precancerous and cancerous oral lesions - Computer-assisted analysis of the oral brush biopsy. Journal of the American Dental Association, 1999. 130(10): p. 1445-1457.

15. Christian, D.C., Computer-assisted analysis of oral brush biopsies at an oral cancer screening program. The Journal of the American Dental Association, 2002. 133(3): p. $357-362$.

16. Burt, J.P.H., Pethig, R., Gascoyne, P.R.C., and Becker, F.F., Dielectrophoretic characterisation of Friend murine erythroleukaemic cells as a measure of induced differentiation. Biochimica et Biophysica Acta (BBA) - General Subjects, 1990. 1034(1): p. 93-101.

17. Huang, Y., Wang, X.-B., Becker, F.F., and Gascoyne, P.R.C., Membrane changes associated with the temperature-sensitive P85gag-mos-dependent transformation of rat kidney cells as determined by dielectrophoresis and electrorotation. Biochimica et Biophysica Acta (BBA) - Biomembranes, 1996. 1282(1): p. 76-84.

18. Gascoyne, P., Pethig, R., Satayavivad, J., Becker, F.F., and Ruchirawat, M., Dielectrophoretic detection of changes in erythrocyte membranes following malarial infection. Biochimica et Biophysica Acta (BBA) - Biomembranes, 1997a. 1323(2): p. 240-252.

19. Gascoyne, P.R.C., Noshari, J., Becker, F.F., and Pethig, R., Use of dielectrophoretic collection spectra for characterizing differences between normal and cancerous cells. Industry Applications, IEEE Transactions on, 1994. 30(4): p. 829-834.

20. An, J., Lee, J., Lee, S., Park, J., and Kim, B., Separation of malignant human breast cancer epithelial cells from healthy epithelial cells using an advanced dielectrophoresis- 
activated cell sorter (DACS). Analytical and Bioanalytical Chemistry, 2009. 394(3): p. 801-809.

21. Broche, L.M., Bhadal, N., Lewis, M.P., Porter, S., Hughes, M.P., and Labeed, F.H., Early detection of oral cancer - Is dielectrophoresis the answer? Oral Oncology, 2007. 43(2): p. 199-203.

22. Yang, L., Arias, L., Lane, T., Yancey, M., and Mamouni, J., Real-time electrical impedance-based measurement to distinguish oral cancer cells and non-cancer oral epithelial cells. Analytical and Bioanalytical Chemistry. 399(5): p. 1823-1833-1833.

23. Broche, L.M., Hoettges, K.F., Ogin, S.L., Kass, G.E.N., and Hughes, M.P., Rapid, automated measurement of dielectrophoresis forces using DEP-activated microwells Electrophoresis, 2011. In Press.

24. Pohl, H.A., Dielectrophoresis the behavior of neutral matter in nonuniform electric fields. 1978: Cambridge University Press.

25. Huang, Y., Holzel, R., Pethig, R., and Wang, X.-B., Differences in the AC electrodynamics of viable and non-viable yeast cells determined through combined dielectrophoresis and electrorotation studies. Physics in Medicine and Biology, 1992. 37(7): p. 1499-1517.

26. Irimajiri, A., Hanai, T., and Inouye, A., Dielectric Theory of Multi-Stratified ShellModel with Its Application to a Lymphoma Cell. Journal of Theoretical Biology, 1979. 78(2): p. 251-269.

27. Hoettges, K.F., Hubner, Y., Broche, L.M., Ogin, S.L., Kass, G.E.N., and Hughes, M.P., Dielectrophoresis-Activated Multiwell Plate for Label-Free High-Throughput Drug Assessment. American Chemical Society, 2008. 80(6): p. 2063-2068. 
28. Hubner, Y., Hoettges, K.F., Kass, G.E.N., Ogin, S.L., and Hughes, M.P., Parallel measurements of drug actions on erythrocytes by dielectrophoresis, using a threedimensional electrode design. Nanobiotechnology, IEE Proceedings-, 2005. 152(4): p. $150-154$

29. Pethig, R., Jakubek, L.M., Sanger, R.H., Heart, E., Corson, E.D., and Smith, P.J.S., Electrokinetic measurements of membrane capacitance and conductance for pancreatic beta-cells. IEE Proceedings Nanobiotechnology, 2005. 152(6): p. 189-193.

30. Gascoyne, P.R.C., Pethig, R., Burt, J.P.H., and Becker, F.F., Membrane changes accompanying the induced differentiation of Friend murine erythroleukemia cells studied by dielectrophoresis. Biochimica et Biophysica Acta (BBA) - Biomembranes, 1993. 1149(1): p. 119-126.

31. Broche, L.M., Labeed, F.H., and Hughes, M.P., Extraction of dielectric properties of multiple populations from dielectrophoretic collection spectrum data. Physics in Medicine and Biology, 2005. 50(10): p. 2267-2274.

32. Morgan, H. and Green, N.G., AC electrokinetics: Colloids and Nanoparticles. First ed. 2002: Research Studies Press Ltd.

33. Pethig, R. and Kell, D.B., The passive electrical properties of biological systems their significance in physiology, biophysics and biotechnology. Physics in Medicine and Biology, 1987. 32(8): p. 933-970.

34. Wang, X.-B., Huang, Y., Gascoyne, P.R.C., Becker, F.F., Hölzel, R., and Pethig, R., Changes in Friend murine erythroleukaemia cell membranes during induced differentiation determined by electrorotation. Biochimica et Biophysica Acta (BBA) Biomembranes, 1994. 1193(2): p. 330-344. 
35. Ratanachoo, K., Gascoyne, P.R.C., and Ruchirawat, M., Detection of cellular responses to toxicants by dielectrophoresis. Biochimica et Biophysica Acta (BBA) Biomembranes, 2002. 1564(2): p. 449-458.

36. Labeed, F.H., Coley, H.M., and Hughes, M.P., Differences in the biophysical properties of membrane and cytoplasm of apoptotic cells revealed using dielectrophoresis. Biochimica et Biophysica Acta (BBA) - General Subjects, 2006. 1760(6): p. 922-929.

37. Partin, A.W., Isaacs, J.T., Treiger, B., and Coffey, D.S., Early Cell Motility Changes Associated with an Increase in Metastatic Ability in Rat Prostatic Cancer Cells Transfected with the v-Harvey-ras Oncogene. Cancer Research, 1988. 48(21): p. 60506053.

38. Nicolas, A.F.v.L., Marc, E.B., and Marc, M.M., Invasive epithelial cells show more fast plasma membrane movements than related or parental non-invasive cells. Cytometry, 1992. 13(1): p. 9-14.

39. Jiang, W.G., Focus on science--Membrane ruffling of cancer cells: A parameter of tumour cell motility and invasion. European Journal of Surgical Oncology, 1995. 21(3): p. 307-309.

40. Becker, F.F., Wang, X.B., Huang, Y., Pethig, R., Vykoukal, J., and Gascoyne, P.R.C., The removal of human leukaemia cells from blood using interdigitated microelectrodes. Journal of Physics D: Applied Physics, 1994. 27(12): p. 2659-2662.

41. Becker, F.F., Wang, X., Huang, Y., Pethig, R., Vykoukal, J., and Gascoyne, P.R.C., Separation of Human Breast Cancer Cells From Blood by Differential Dielectric Affinity. 1995. p. 860-864. 
42. Bhoopathi, V., Kabani, S., and Mascarenhas, A.K., Low Positive Predictive Value of the Oral Brush Biopsy in Detecting Dysplastic Oral Lesions. Cancer, 2009. 115(5): p. 1036-1040.

43. Epstein, J.B., Silverman Jr, S., Epstein, J.D., Lonky, S.A., and Bride, M.A., Analysis of oral lesion biopsies identified and evaluated by visual examination, chemiluminescence and toluidine blue. Oral Oncology, 2008. 44(6): p. 538-544. 
Figure I: Images of negative and positive DEP occurring in a DEP-microwell. Images taken through a DEP-microwell containing cell suspensions before $(a, c)$ and after $(b, d)$ the application of an electric field for 60 seconds. In figures (a) and (b) the energising frequency was $6.3 \mathrm{kHz}$, resulting in negative DEP, whereas in (c) and $(d)$ the energising frequency was $250 \mathrm{kHz}$ resulting in positive dielectrophoresis. In both cases a depletion band forms around the outer part of the well as cells move towards or away from the well perimeter;; however; in negative DEP cells are concentrated in the centre which becomes darker, whereas in positive DEP the collect at the well periphery (out of sight), resulting in cell depletion in the centre which becomes brighter.

\section{Figure II: An example of typical electrophysiological spectra produced using the DEP-microwell.}

A typical spectrum of oral keratinocytes can be produced when the change in light intensity in the region of interest in the DEP-microwell is plotted against frequency. The spectrum is negative at low frequencies, where cells are repelled from the electrodes; at higher frequencies the spectrum becomes positive, where cells are being attracted to the electrodes. The spectrum gradually increases until a plateau stage is reached. At higher frequencies still, the spectrum begins to decrease.

\section{Figure III: Effective Membrane Capacitance of Normal, Dysplastic and}

\section{Cancerous Oral Keratinocytes.}

The effective membrane capacitance of primary normal (HOK), dysplastic (DOK) and OSCC (H357 and H157) oral keratinocytes were derived by fitting lines of best fit to dielectric spectra and applying the 'single shell model' [24, 25]. Error bars denote the standard deviation (SD). Each experiment was repeated at least five times. Asterisks denote a statistically significant difference $(\mathrm{P}<0.05, \quad \mathrm{P}<0.01)$. 
Figure IV: Cytoplasmic Conductivity of Normal, Dysplastic and Cancerous Oral Keratinocytes.

The cytoplasmic conductivity of primary normal (HOK), dysplastic (DOK) and OSCC (H357 and H157) oral keratinocytes were derived by fitting lines of best fit to dielectric spectra and applying the 'single shell model' [24, 25]. Error bars denote the standard deviation (SD). Each experiment was repeated at least five times. Asterisks denote a statistically significant difference ( $\star \star * \gtrless<0.05, \quad P<0.01)$.

\section{Figure V: DEP Scatter Plot}

The cytoplasmic conductivity and effective specific membrane capacitance of primary normal (HOK), dysplastic (DOK) and OSCC (H357 and H157) oral keratinocytes were derived by fitting lines of best fit to dielectric spectra and applying the 'single shell model' [24, 25]. Each experiment was repeated five times (some points overlap). 
Figure I

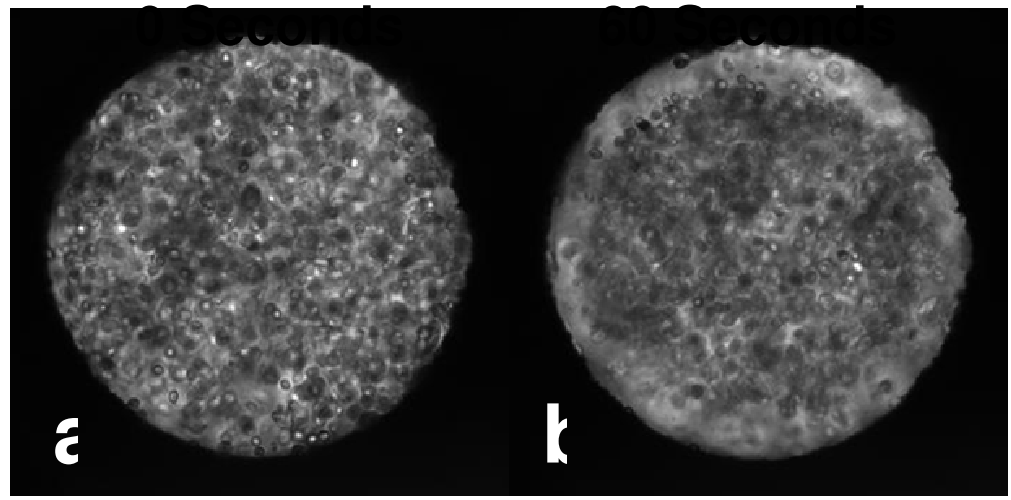

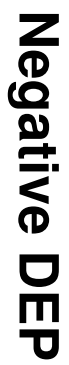

Figure II
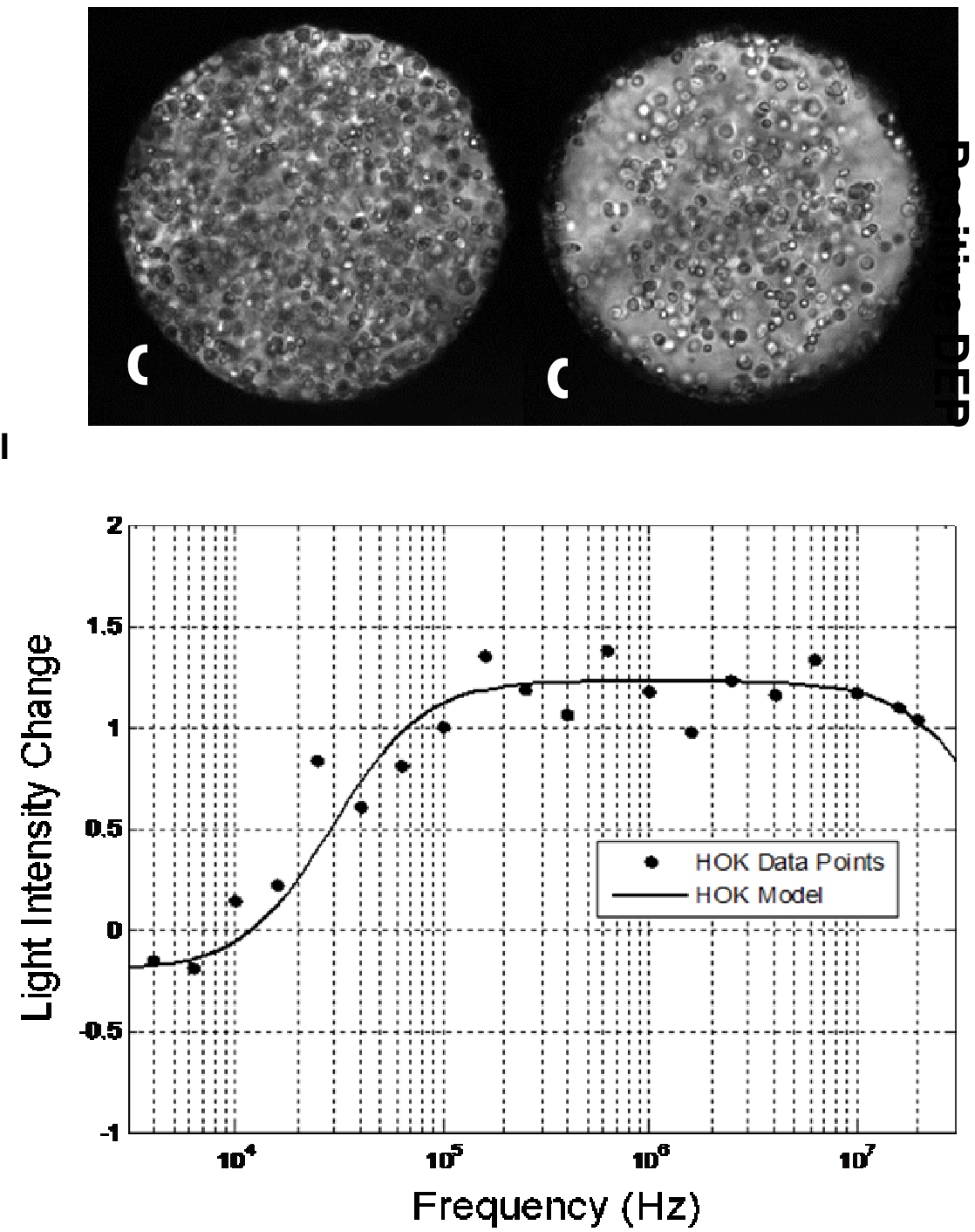

Figure III 


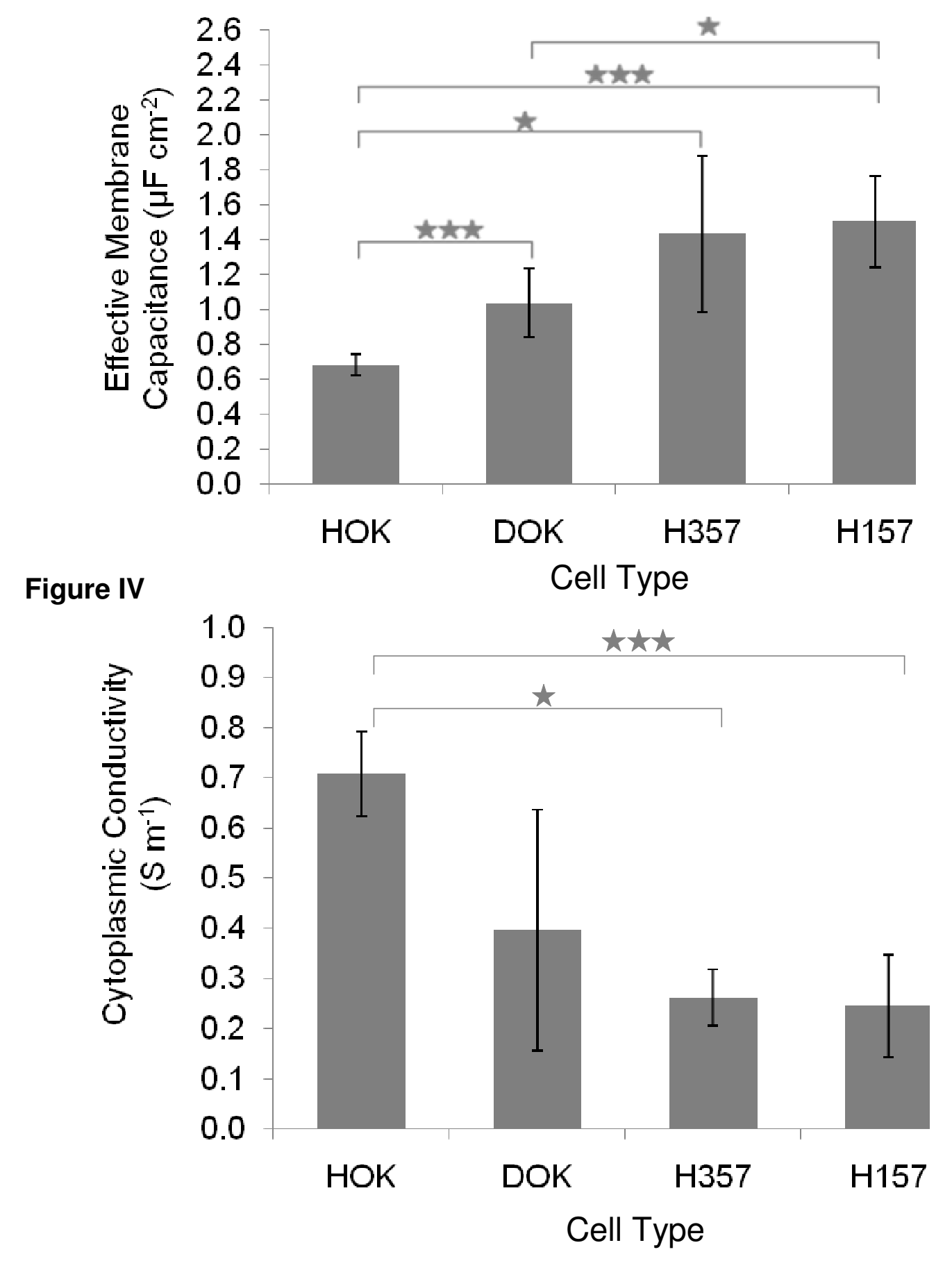

Figure V 


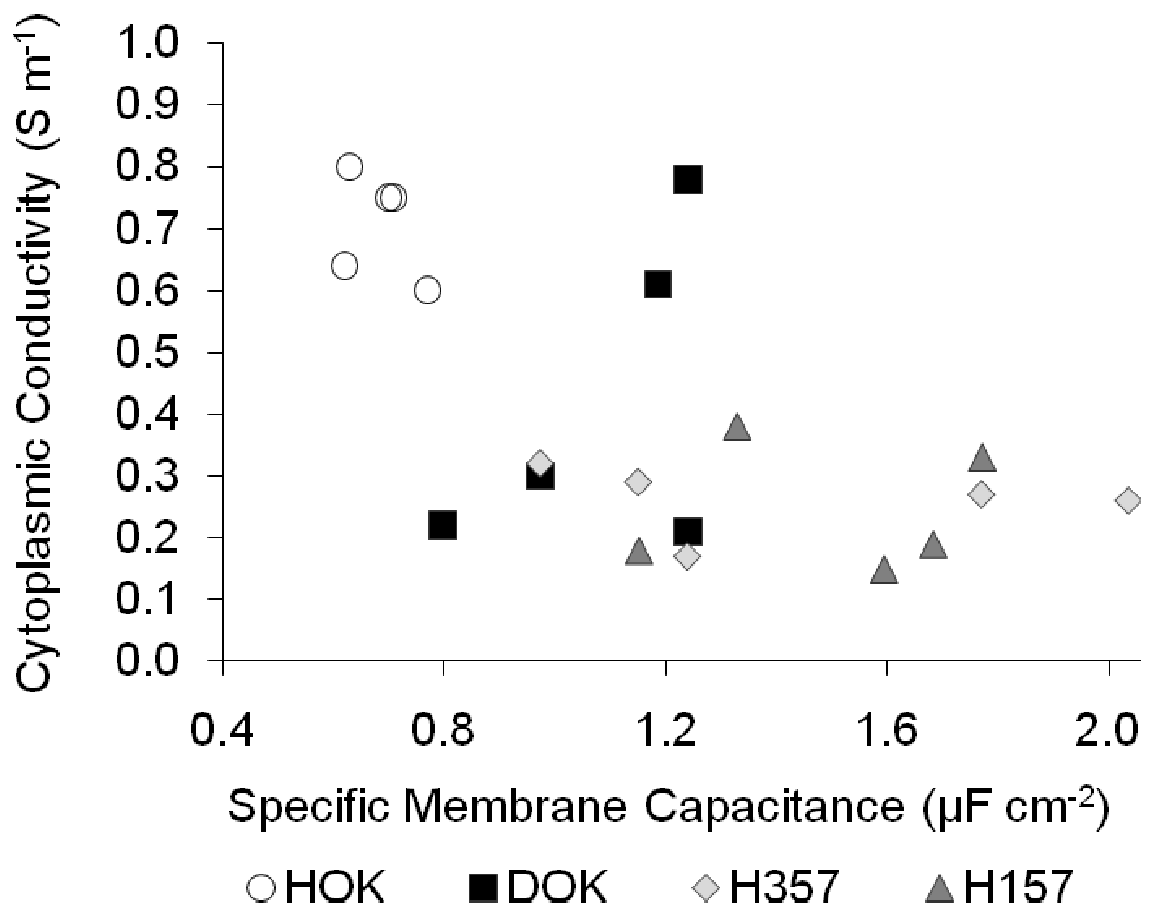

